\title{
Preparation and Evaluation of Proliposomes Containing Clotrimazole
}

\author{
Mei-Ying Ning, ${ }^{*, a, b}$ Ying-Zhi Guo, ${ }^{a, b}$ Huai-Zhong PAN, ${ }^{b}$ He-Ming Yu, ${ }^{b}$ and Zhong-Wei Gu ${ }^{b}$ \\ ${ }^{a}$ Chinese Academy of Medical Science \& Peking Union Medical College; No. 9, Dongdan No. 3 avenue, Beijing 100730, \\ P. R. China: and ${ }^{b}$ Centre of Drug Controlled Release Research, National Research Institute for Family Planning; No. 12, \\ Da Hui Si Haidian District, Beijing 100081, P. R. China. $\quad$ Received January 5, 2005; accepted March 3, 2005
}

\begin{abstract}
Clotrimazole (CT)-containing proliposomes were prepared by penetrating an ethanol solution of CT and Egg phosphatidylcholine (PC) into microporous sorbitol particles, followed by vacuum evaporation of the solvent. As a result, CT proliposomes with free-flowing flowability were obtained. On contact with water, the proliposomes were rapidly converted into a liposomal dispersion, in which a certain amount of CT was entrapped by the liposomes. The result in scanning electronic micrograph confirmed the formation of liposomes structures from proliposomes, and the particles revealed round or ellipse. The ratio of drug to total lipid, ratio of PC to cholesterol and ratio of lipid to sorbitol affected the entrapment efficiency (EE\%). The EE\% of optimized formulation (CT $10 \mathrm{mg}, 0.1 \mathrm{~g}$ total lipid, $\mathrm{PC} / \mathrm{CH}$ ratio is $60: 40$ and $1 \mathrm{~g}$ sorbitol) in this investigation was $96.2 \pm 1.5 \%$. The proliposomes system can provide sustaining release in simulated vaginal fluid at $37 \pm 1^{\circ} \mathrm{C}$ for $24 \mathrm{~h}$. In-vivo performance of blank proliposomes, a physical mixture of sorbitol and drug, clotrimazole proliposomes and commercial ointment formulation were evaluated using antifungal activity test. At 7 d post-dose, the c.f.u. of $C$. albicans decreased in proliposomes-treated groups than ointment and the physical mixture ( $t$-Student, $p<0.05$ ). The results indicated that CT-containing vaginal proliposomes prolonged drug release and may increase amount of drug retention into the mucosa to result in more antifungal efficacy. In addition, CT-proliposomes did not affect the morphology of vaginal tissues. Therefore, the dosage form might be further developed for safe, convenient, and effective treatment of vaginal candidasis with reduced dosing interval.
\end{abstract}

Key words proliposome; clotrimazole; vaginal drug delivery

Clotrimazole (CT), which is an imidazole derivative, is widely and effectively used for the treatment of vulvovaginal candidiasis. ${ }^{1)}$ Unfortunately, oral use of CT is unacceptable due to the severe side effects. Thus, topical administration of $\mathrm{CT}$ is recommended. However, it is limited by its very low water solubility resulting in the essential to incorporate $\mathrm{CT}$ into a suitable vehicle. Commercially conventional $\mathrm{CT}$ vaginal delivery systems, such as creams, foams, and gells, are considered to reside for a relatively short period of time at the targeted site. The entrapment of drug in vesicles is viewed to help in the localized delivery of the drug and an improved solubility and availability of the drug at the site will reduce the dose.

As can be known, liposomes are starting to be widely investigated in topical applications for the skin, ${ }^{2-4)} \mathrm{oral}^{5)}$ and vaginal diseases. ${ }^{67)}$ Liposomes have been shown to enhance the penetration of vesicle-bound-drugs into the skin, after topical application, acting as a "drug localizers," with low systemic absorption of the drugs, in comparison to other galenical formulation, resulting in less drug side-effect and sustained drug releasing. ${ }^{2)}$

But liposomes still have physical stability problems associated with the aqueous suspension such as aggregation, fusion limit their shelf life. Concern on these problems, these stability problems can be avoided by formulating liposomes as proliposomes. Proliposomes are dry, free-flowing products, which, on addition of water, disperse to form a liposomal suspension. ${ }^{8)}$ Thus, it can be fabricated in various dosage forms including tablets and capsules which were more convenient for vaginal administration than liposomes solution. In addition, proliposomes have a number of advantages over liposomes. For example, they tolerate sterilization by ultraviolet and are much more stable physicochemically. Proliposomes offer a versatile liposomes delivery concept with potential for use with a wide range of active compounds and various drug administration routines. ${ }^{9-15)}$

In present study it was to investigate the feasibility of proliposomes to formulate the vaginal administration of CT. Proliposomes are formed by penetrating organic solution of $\mathrm{CT}$ and phosphatidylcholine (PC) into microporous sorbitol particles, followed by vacuum evaporation of the solvent method. Formulations composed of egg phospholipid, cholesterol compositions have been characterized by encapsulation efficiency. In addition, we are investigating the optimized resultant liposomes in vitro release study. Furthermore, we describes an attempt to achieve the antifungal activity more efficiency followed by a prolonged delivery of CT via a vaginal application of appropriate dosage forms of CT. A physical mixture of CT and sorbitol was also prepared and compared for antifungal efficiency with proliposomes following vaginal application in rats. Further, proliposomes system was evaluated by tolerability on tissue level in rat.

\section{Experimental}

Materials Clotrimazole (CT) and Egg phospholipids (PC) (>98\%) were the generous gifts of Xi'an Libang Liposomes Pharmaceutical Company. Cholesterol (Chol) and sorbitol were brought from Sigma Company. Cellulose nitrate membrane filters $(0.22 \mu \mathrm{m}$, Whatman, Majdstone, U.K.); Dialysis bag (cut off 12000 - 14000) was purchased from Sigma Company. Acetate buffer ${ }^{7}(\mathrm{pH} 4.5)$ was made of $0.2 \mathrm{M} \mathrm{CH}_{3} \mathrm{COONa}(430 \mathrm{ml})$ and $0.2 \mathrm{M}$ $\mathrm{CH}_{3} \mathrm{COOH}(570 \mathrm{ml})$. All other reagents used in the study were of analytical grade and commercially available.

Preparation of CT Proliposomes CT-containing proliposomes were prepared by penetrating an ethanol solution of $\mathrm{CT}$ and PC into microporous sorbitol particles, followed by vacuum evaporation of the solvent. Precisely, a $50 \mathrm{ml}$ round-bottom flask containing $1 \mathrm{~g}$ of sorbitol (mean diameter of $180-400 \mu \mathrm{m}$ ) was attached to the rotary evaporator. Formulations containing different compositions with PC, cholesterol were dissolved in ethanol solution and were introduced into the round-bottom flask on the rotary evaporator by sequential powder. During the spraying period, the rate of application was controlled so that the powder bed of sorbitol did not become overly wet. The evaporator was then evacuated and the rotating flask was lowered into a water bath at $35^{\circ} \mathrm{C}$. The flask was rotated in the water bath under vacuum for $15-20 \mathrm{~min}$ or until sorbitol appeared to be dry, and another aliquot 
of lipid solution had been applied. After addition of the final aliquot, evaporation was continued until the powder was completely dry. The material was further dried in a desiccator under vacuum at room temperature overnight. This dry preparation is referred to as "proliposomes" and was used for preparations and for further study on powder properties.

Proliposomes-drived liposomal dispersions were obtained by hydrating the proliposome $(10 \mathrm{mg})$ preparation with $35^{\circ} \mathrm{C}$ distilled water $1 \mathrm{ml}$ and vortexes mixing for a while till no residual substance in the bottom of vial. The resulting liposome dispersion was used for the determination of the entrapment efficiency and morphological studies. The entrapment efficiency of CT in liposomes was used as a criterion for the evaluation of the tested formulations.

Scanning Electron Microscopy (SEM) The surface morphology of proliposomes and liposomes from prolipsomes was examined by SEM after coating them with platinum in an ion sputter coater (Eilo IB-5, Japan), and was photographed using a HITACHI scanning electron microscope (HITACHI S-2500, Japan).

Drug-Entrapment Efficiency The entrapment liposomes were separated by dialysis. Dialysis was applied to separate unentrapped drugs from hydration liposomes containing drugs. The procedure was as follows: sample of liposome suspension was placed in a tube (MW cut off 12000 14000) and extensively dialysed against the buffer solution for $5 \mathrm{~h}$. One milliliter of clotrimazole liposomal suspension each of which before and after dialysis was diluted and adjusted to volume with methanol in a $10-\mathrm{ml}$ volumetric flask, and the amount of drug was determined by HPLC. The HPLC system consisted of Gold Nouveau software workstation, a Beckman 126 NM solvent delivery system, Beckman 508 autosampler with a 100- $\mu$ l loop, and Beckman 168 NM PDA detector. The column used was Beckman C18 dp $5 \mu \mathrm{m}, 4.6 \mathrm{~mm} \times 25 \mathrm{~cm}$ (Beckman, U.S.A.). The mobile phase consisted of methanol and $\mathrm{H}_{2} \mathrm{O}(\mathrm{pH} 3.0)(95: 5, \mathrm{v} / \mathrm{v})$. The flow rate was $0.8 \mathrm{ml} / \mathrm{min}$. The chromatogram was monitored at a wavelength of $220 \mathrm{~nm}$.

The regression equation for clotrimazole content $(\mu \mathrm{g} / \mathrm{ml})$ in methanol ranging from 10 to $500 \mu \mathrm{g} / \mathrm{ml}$ was: $C=18013 A-28.537,\left(R^{2}=0.9995\right)$, where $C(\mu \mathrm{g} / \mathrm{ml})$ and $A$ represented the concentration and peak area of clotrimazole, respectively. The mean recovery was $98.50 \pm 1.73(n=3)$. The precision assay showed that relative standard deviations within $1 \mathrm{~d}$ and among every other day were all below $2 \%$. This method was validated in terms of specificity, linearity and reproducibility. The limit of quantification was $1 \mu \mathrm{g} / \mathrm{ml}$. The exact amounts of clotrimazole were using the calibration curve. tion:

Entrapment efficiency (EE\%) could be achieved by the following equa-

\section{$\mathrm{EE} \%=$ content of $\mathrm{CT}$ in post-dialyzed liposomes/content of $\mathrm{CT}$ in pre-dialyzed liposomes $\times 100 \%$}

In present study, dialysis method was applied in entrapment efficiency determination. Recovery of drug was determined for all samples and was between $94.5 \%$ and $96.2 \%$ of the amount taken into preparation.

Angle of Repose Because proliposomes are a dry powder, further processing is possible. To provide convenient unit dosing, the present proliposomes powder may be processed to make tablets or capsules for vaginal drug delivery. Angle of repose measurements indicated that the fluidity of proliposomes dry powder is equal to or better than that of sorbitol powder, so further processing of proliposomes powder should be straightforward. The angle of repose of dry proliposome powder was measured by a funnel method.

Release Study In order to investigate the formulations release profile in simulated physiological condition, the EE\% change of liposomes were tested in PBS and acetic acid buffer $(\mathrm{pH} 4.5)$ at $37 \pm 1^{\circ} \mathrm{C}$. We chose the formulation: CT-proliposomes using $0.1 \mathrm{~g}$ total lipid, $1 \mathrm{~g}$ sorbitol, $\mathrm{PC} / \mathrm{CH}$ ratio was $60: 40$. The drug release percent from vesicles results are shown in Fig. 3. Briefly, dialysis tubes containing $2.5 \mathrm{ml}$ of CT liposomes were placed into a flask containing $125 \mathrm{ml}$ of vaginal fluid or $\mathrm{PBS}(\mathrm{pH}, 7.4)$ at $37^{\circ} \mathrm{C}$. The bag was secured with two knots at each end and the air spaces minimized as much as possible. These flasks were placed in a shaker (HZQ-C, Har'er bin, China) and shaken at $100 \mathrm{rpm}$ at a temperature of $37^{\circ} \mathrm{C}$. The samples from liposomes were withdrawn at definite time intervals; the residual amount of drug in vesicles was determined as described mentioned above.

Antifungicidal Efficacy Studies A major problem in studies of vaginal absorption in rodent species is the variable structure of the vaginal epithelium at different stages of the oestrous cycle. In order to standardize the thickness of the vaginal epithelium, the animals used in the present study were ovariectomised. Ovariectomised female Spraue-Dawley rats, $200 \pm 10 \mathrm{~g}$ (body weight), receiving subcutaneous administration of estradiol benzoate $(25 \mathrm{mg} / \mathrm{kg})$ every $2 \mathrm{~d}$ during the experiment, were selected and housed in individual cages and received food and water ad libitum. The animals were infected by intravaginal inoculation of C. albicans (CMCC(B) 98001) suspended in sterile saline containing $10^{8}$ c.f.u. $/ \mathrm{ml}$. A vaginal smear was taken $2 \mathrm{~d}$ after the challenge to confirm the establishment of infection.

Female rats were randomly divided into 4 groups (4 in each group). Group 1, vaginal administration of proliposomes without drug, as negative control; group 2, vaginal administration of a physical mixture of sorbitol and drug $(25 \mathrm{mg} / \mathrm{kg})$; group 3, vaginal administration of commercial CT ointment; group 4, vaginal administration proliposomes $(25 \mathrm{mg} / \mathrm{kg})$.

The rats were fasted for $24 \mathrm{~h}$ prior to the experiments. Ovariectomised rats were anaesthetized by intraperitoneal injection of $60 \mathrm{mg} / \mathrm{kg}$ pentobarbitone sodium (Beijing Chemical Reagents Company, Beijing). The proliposomes powder was inserted into the vaginal tract through a plastic tube at a CT of $25 \mathrm{mg} / \mathrm{kg}$ once a day for 3 consecutive days starting $24 \mathrm{~h}$ after challenge (day 0). For control experiments, blank proliposomes and the physical mixture of sorbitol with free CT was similarly administrated, the commercial CT ointment were applied into the vaginal tract of rats at a CT dose of $25 \mathrm{mg} / \mathrm{kg}$ using a stomach sondle needle. For the vaginal colony counts, an analysis of variance was done on the $\log _{10}$ colony counts for each day of vaginal culture and each drug formulation from days 4 to 7 . Vaginal lavage samples were collected with $100 \mu \mathrm{l}$ saline by washing the fluid three times up and down in the vagina. The fluid was then plated onto sabouraud dextrose agar and incubated for $48 \mathrm{~h}$ at $37 \pm 1{ }^{\circ} \mathrm{C}$ and c.f.u. values were recorded.

Morphology Study of Vaginal Tissues after Application of CT-Proliposomes Oophorectomized female Sprague-Dawley rats weighing $200 \pm 10 \mathrm{~g}$ were used after a recovery period of at least $7 \mathrm{~d}$. CT-proliposomes were administered into the vagina of the rats at a CT dose of $25 \mathrm{mg} / \mathrm{kg}$, the vaginal tissues of the blank sorbitol treated rats (A) and CT-proliposomes rats (B) were isolated, fixed in $10 \%$ neutral carbonated-buffered formaldehyde, embedded in paraffin, and cut into slices. After hemotoxylin-eosin staining, the slices were observed under a light microscope.

Data Analysis Data were analyzed statistically by the one way ANOVA analysis using the Microsoft Excell 2000 and by the $t$-Student test (level of significance for $p<0.05$ ).

\section{Results and Discussion}

Microscopic Appearance Scanning electron microscopy of uncoated sorbitol and dry proliposomes powder (Fig. 1a) reveals that there appears to be a slight difference on the appearance of the surfaces. The powder in (Fig. 1b) appears to be smoother and to have fewer "fine feature" such as whiskers and sharp corners.

The morphology of the resultant liposome vesicles was evaluated. Figure 2 shows the shape of the resultant liposomes entraping with the drug clotrimazole. It was evident that the particles investigated revealed round or ellipse.

Effect of CT Content With the total concentration of lipid kept constant at $0.1 \mathrm{~g}$, the ratio of PC to Chol was $6: 4$
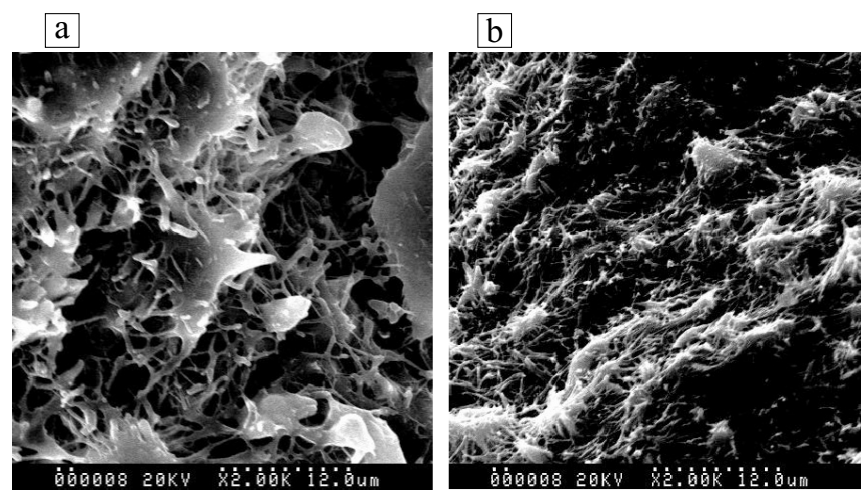

Fig. 1. Scanning Electron Micrograph of Uncoated Sorbitol and Egg Phospholipid: Cholesterol : Clotrimazole Proliposomes $(\times 2000)$

CT proliposomes formulation: CT $10 \mathrm{mg}, 0.1 \mathrm{~g}$ total lipid, $\mathrm{PC} / \mathrm{CH}$ ratio is $60: 40,1 \mathrm{~g}$ sorbitol. 


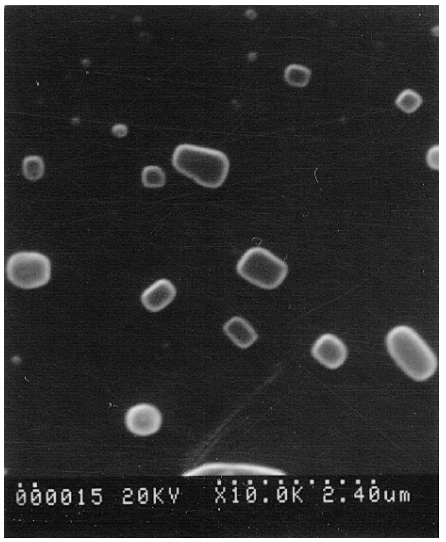

Fig. 2. Scanning Electron Micrograph of Resulted Liposomes from Proliposomes

CT proliposomes formulation: CT $10 \mathrm{mg}, 0.1 \mathrm{~g}$ total lipid, $\mathrm{PC} / \mathrm{CH}$ ratio is $60: 40,1 \mathrm{~g}$ sorbitol.

Table 1. Effect of Different CT Amount on Entrapment Efficiency $(\%, n=3)$ of CT Proliposomes Using $0.1 \mathrm{~g}$ Total Lipid, $1 \mathrm{~g}$ Sorbitol

\begin{tabular}{cc}
\hline \hline CT amount $(\mathrm{mg})$ & Entrapment efficiency $(\%)$ \\
\hline 2.5 & $96.2 \pm 1.2$ \\
5.0 & $95.3 \pm 3.6$ \\
10 & $96.2 \pm 1.5$ \\
15 & $75.8 \pm 2.5$ \\
20 & $55.6 \pm 2.5$ \\
\hline
\end{tabular}

$\mathrm{PC} / \mathrm{CH}$ ratio is $60: 40$.

to investigate the effect of drug content on $\mathrm{CT}$ entrapment efficiency (Table 1). The concentration of CT was changed to verify that the drug was significantly altering the behavior of the liposomes preparation over $10 \%(\mathrm{~g} / \mathrm{g})$ of total lipid, and crystalline drug existing in the preparation under an optical microscope, which might be accounted to the saturation of lipid domains with reference to drug where low PC content provides limited entrapment capacity.

The entrapment efficiency of CT showed no significant difference, remaining at about $95 \%$ when CT content is below $3 \mathrm{~mol} \%$ of total lipid. The result supported the statement made by Payne ${ }^{16)}$ that the proliposome system is ideally suited for lipophilic actives, where after suitable optimization, the majority of drug partitions into the liposomal lipid phase.

Effect of PC-to-Chol Ratio The percentage drug entrapment efficiencies of $\mathrm{CT}$ proliposomes prepared at various amount ratios of $\mathrm{PC}$ to Chol are shown in Table 2, which reveals that incorporation of more Chol would yield higher EE (\%) at a constant molar ratio range. The content of Chol is one of the important parameters in the design of proliposomal preparations, as well as liposomes. Cholesterol does not itself form the bilayer structure but it can be incorporated into the phospholipid bilayers. Since it is amphipathic, Chol could be inserted into the membrane with its hydroxyl group oriented toward the aqueous surface and the aliphatic chain aligned parallel to the acyl chains in the center of the bilayer. But, when the Chol content is over a range, it might lower the partitioning of drug molecules to the bilayer membrane, the degree of encapsulation would decrease. ${ }^{17)}$ As can be seen, the entrapment efficiencies of all proliposomal formulations
Table 2. Effect of PC/CH Ratio on CT Entrapment Efficiency $(\%, n=3)$ of CT Proliposomes Using $10 \mathrm{mg}$ CT

\begin{tabular}{cc}
\hline \hline Molar ratio PC/CH ratio & Entrapment efficiency (\%) \\
\hline $90: 10$ & $70.2 \pm 2.5$ \\
$80: 20$ & $89.1 \pm 2.5$ \\
$70: 30$ & $90.1 \pm 1.5$ \\
$60: 40$ & $95.2 \pm 3.6$ \\
$50: 50$ & $93.1 \pm 1.9$ \\
$30: 70$ & $73.1 \pm 2.1$ \\
\hline
\end{tabular}

Table 3. Angle of Repose of Dry Proliposome Powder

\begin{tabular}{lccc}
\hline \hline & \multicolumn{3}{c}{ Sorbitol } \\
\cline { 2 - 4 } & Sorbitol & Proliposome & Proliposome $(2 \times)^{a)}$ \\
\hline $\begin{array}{c}\text { Preparation } \\
\text { angle }\end{array}$ & $43.19 \pm 0.75$ & $34.66 \pm 2.10$ & $37.65 \pm 1.07$ \\
\hline
\end{tabular}

a) The mass of sorbitol was doubled, but the mass of lipid was kept constant.

were found to be significantly enhanced with the increasing Chol content $(p<0.05)$ when PC-to-Chol ratio is lower than $50: 50$. It was observed Chol crystal when PC-to-Chol ratio was higher than $5: 5$. The enhancing effect of Chol on the entrapment efficiency may be attributed to the rigidifying effect in the fluid crystal state, facilitating the complete formation of the vesicles with the bilayer-bound drug during the process of proliposomes hydration.

Effect of Total Lipid-to-Sorbitol Ratio The concentrtion of sorbitol has no measurable effect on $\mathrm{EE} \%$ of $\mathrm{CT}$, based on comparison of total lipid: sorbitol ratios from $1: 10$ to $1: 20$. However, from the point of preparation, it is difficult to prepare the proliposomes when the total lipid-to-sorbitol ratio is higher than $1: 10$. Because only a very small volume of the solution of membrane-forming components can be introduced and sprayed onto the limited amount of sorbitol each time, the spraying-evaporating process becomes much time consuming. Because a higher sorbitol concentration did not improve the formation, $1: 10$ sorbitol was used for the formulation of proliposomes.

Angle of Repose Results of measurement of the angle of repose of proliposome powder and pure sorbitol are summarized in Table 3, and indicate that the angle of repose of dry proliposomes powder is smaller than that of pure sorbitol. This is consistent with the scanning electron microscopic observation of proliposome powder, in which it was observed that the proliposomes surface was smoother. If the proportion of sorbitol to lipid is increased, the angle of repose of dry proliposome powder increases slightly, more closely approaching the angle measured for pure sorbitol.

Release Study In order to evaluate the controlled release potential of the investigated formulations, the diffusion of clotrimazole from the proliposomes was investigated over $24 \mathrm{~h}$. Each sample was analyzed in triplicate. The results are shown in Fig. 3.

The release rate of CT proliposomes in acetic buffer $(\mathrm{pH}$ 4.5) are faster than from those in PBS, which is to be expected due to the dissociation constants of the $\mathrm{CT}$ that is a weak base $\left(\mathrm{p} K_{\mathrm{a}}, 4.7\right)$. Within approximately $8 \mathrm{~h}$, the CT release in acetic buffer is about $35 \%$, in PBS is about $20 \%$. After $24 \mathrm{~h}, \mathrm{CT}$ release from liposomes in acetic buffer is 
$45 \%$, in PBS had only reached about $25 \%$.

Antifungicidal Efficacy Studies Experimentally induced vaginal candidiasis in this model is hormonal dependent, making treatment with estradiol valerated essential. Positive/negative cultures taken immediately before challenge were all negative indicating no prechallenge $C$. albicans vaginal colonization. C. albicans challenge inocula were verified by serial plating on sabouraud dextrose agar to be $5.0 \times 10^{5}$ blastospores for study. This gave a well-established infection in all challenged animals (Table 4) with a range of 4.89 to $5.05 \log _{10}$ c.f.u. $/ \mathrm{ml}$ on day 0 prior to treatment. All animals had well established infections on day 0 with very little variability. The blank 1 (without drug proliposomes)treated controls maintained an excellent level of infection throughout the studies (day 7). This was an indication and an appropriated challenge inoculum. In addition, drug dosages and clinical dosing regimens vary greatly when comparing with commericially available vaginal preparations. These differences make direct comparisons between compounds difficult. One way to compare various formulations is to subject them to the same dosing regimen and allow the concentration of drug to be the variable.

In study, formulations including the control and standard ointment (Table 4 ) reduced the number of yeasts in the vaginas on day $4,5,6(p<0.05)$ compared to day 0 . But, there is difference between proliposomes and mixture of sorbitol and drug or commercial ointment on observed day 5, 6, 7. On day 4 , the ointment group deceased greatest in all formulations, which resulted from drug from ointment more rapidly than others, but did not significantly reduce the number of yeasts in the vaginas days $7(p<0.05)$ when compared to day 0 . However, the proliposomes show difference result compared to the ointment on day 7 , which suggested a longer term reduction of yeasts in the vaginal with proliposomes. As Table 4 also showed no significant differences from the ointment and mixture were detected on day 7 which might be the

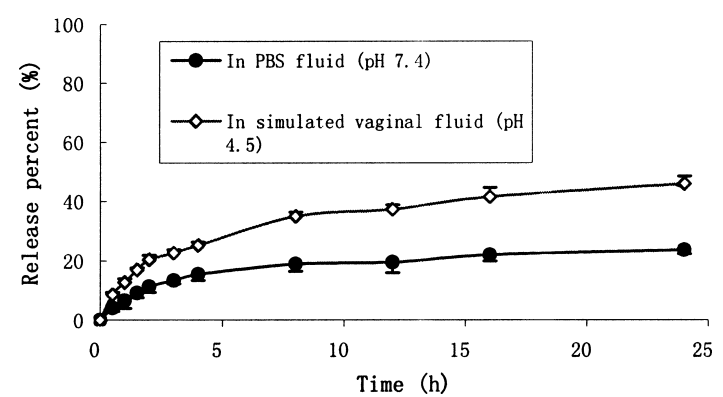

Fig. 3. Release of Clotrimazole in PBS Solution and in Simulated Vaginal Fluid for Proliposomes-Derived Liposomes

The values denote the mean of three preparations \pm S.D. higher drug vaginal mucosa retention, creation of reservoir effect for drug in mucosa due to deposition of other components of resultant liposomes from proliposomes hydration contacting with vaginal fluid with drug into the mucosa and thereby increasing the drug retention capacity into the mucosa. The significantly higher mucosa retention of the liposomes drug resulting in higher partitioning of the drug into the mucosa may be responsible for prolonged and enhanced antifungal activity.

Tolerability of Clotrimazole CT-Proliposomes in Tissue Level CT-proliposomes did not alter the morphology of vaginal tissues. Figure 4 shows the histopathology of the vaginal mucosa after intravaginal application of CT-containing proliposomesl powder. As compared to the control with no treatment, the CT-proliposomes-treated group showed no visible sign of inflammation or necrosis. CT-proliposomes did not affect the morphology of vaginal tissues, which indicated such drug delivery systems are safety for vaginal delivery.

\section{Conclusion}

The result confirmed the formation of liposomes structures from proliposomes, and the particles revealed round or ellipse. Compared with conventional liposomes, the preparation of liposome dispersions from these proliposomes is much more convenient. Apparently due to the great surface area of the lipid film that form on the surface of sorbitol, the hydration of the proliposomes and the formation of the liposome dispersion is very easy. Because the lipid is all coated on the soluble sorbitol, there is little risk of material loss. The hydration of proliposomes progressed sufficiently rapidly as to be evident at times as short as $30 \mathrm{~s}$ after contact with water, suggesting a progressive and rapid conversion to liposomes on contact with physiological fluids (such as vaginal fluid) in the body. The proliposomes system can provide
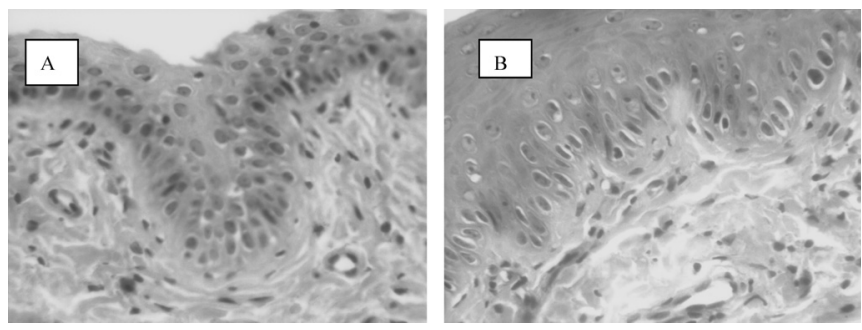

Fig. 4. Morphology of Vaginal Tissues after Application of Sorbitol and CT-Proliposomes Powder were Administered into the Vagina of the Rats at a CT Dose of $25 \mathrm{mg} / \mathrm{kg}$

The vaginal tissues of the blank sorbitol treated rats (A) and proliposomes powdertreated rats (B) were isolated, fixed in $10 \%$ neutral carbonated-buffered formaldehyde, embedded in paraffin, and cut into slices. After hemotoxylin-eosin staining, the slices were observed under a light microscope $(\times 160)$.

Table 4. Antifungal Efficacy of the Prepared Proliposomes (Concentrations of C. albicans $\log$ c.f.u./ml) $(n=4)$

\begin{tabular}{clll}
\hline \hline Time (d) & Blank & Control & Standard \\
\hline 0 & $4.88 \pm 0.23$ & $5.01 \pm 0.33$ & $4.93 \pm 0.54$ \\
4 & $4.91 \pm 0.52$ & $1.71 \pm 0.23^{*}$ & $1.03 \pm 0.14^{*}$ \\
5 & $4.93 \pm 0.57$ & $1.83 \pm 0.25^{*}$ & $1.81 \pm 0.25^{*}$ \\
6 & $4.92 \pm 0.43$ & $3.95 \pm 0.31^{*}$ & $2.93 \pm 0.28^{*, * *}$ \\
7 & $4.96 \pm 0.26$ & $4.84 \pm 0.27$ & $4.83 \pm 0.28$ \\
\end{tabular}

Blank: proliposomes without drug; control: a physical mixture of sorbitol and drug; standard: commercial clotrimazole ointment. $* p<0.05$; compared to the day 0 ; $* * p<0.05$, compared to the mixture control; *** $p<0.05$, compared to the standard. 
sustaining release in simulated vaginal fluid at $37 \pm 1{ }^{\circ} \mathrm{C}$ for $24 \mathrm{~h}$. In-vivo performance of blank proliposomes, a physical mixture of sorbitol, clotrimazole proliposomes and marketed ointment were evaluated using antifungal activity test. At $7 \mathrm{~d}$ post-dose, the c.f.u. of $C$. albicans decreased in proliposomes-treated groups than ointment and the physical mixture ( $t$-student, $p<0.05)$. The results indicated that CT-containing vaginal proliposomes prolonged drug release and may increase amount of drug retention into the mucosa to result in more antifungal efficacy. In addition, CT-proliposomes did not affect the morphology of vaginal tissues. Therefore, the dosage form might be further developed for safe, convenient, and effective treatment of vaginal candidasis with reduced dosing interval.

\section{Reference}

1) Ceschel G. C., Maffei P., Lombardi Borgia S., Drug Dev. Ind. Pharm., 27, 541-547 (2001).

2) Kim M. K., Chung S. J., Lee M. H., Cho A. R., Shim C. K., J. Control. Rel., 46, 243-251 (1997).

3) Valenta C., Wanka M., Heidlas J., J. Control. Rel., 63, 165-173 (2000)

4) Schmid M. H., Korting H. C., Crit. Rev. Ther. Drug Carrier Syst., 11,
97-118 (1994).

5) Farshi F. S., Ozen A. Y., Ercan M. T. J., Microencapsul., 13, 537-544 (1996).

6) Pavelié Ž., Škalko N., Jalšenjak I., Eur. J. Pharm. Science, 8, 345351 (1999).

7) Pavelié Ž., Skalko N., Jalšenjak I., Int. J. Pharm., 219, 139-149 (2001).

8) Payne N. I., Timmins P., Ambrose C. V., Ward M. D., Ridgway F. J., Pharm. Sci., 75, 325-329 (1986).

9) Ahn B. N., Kim S. K., Shim C. K., J. Microencapsul., 12, 363-375 (1995).

10) Katare O. P., Vyas S. P., Dixit V. K., J. Microencapsul., 8, 1 -7 (1991).

11) Deo M. R., Sant V. P., Parekh S. R., Khopade A. J., Banakar U. V., J. Biomater Appl., 12, 77-88 (1997).

12) Wang J. Y., Xu Y. R., Huang K., Sun L. Y., J. Pharm. Pharmacol., 47, 1053-1054 (1995).

13) Jung B. H., Chung B. C., Lee M. H., Shim C. K., J. Control. Rel., 66, 73-79 (2000).

14) Betageri G. V., Yatvin M. B., Cell Mol. Biol. Lett., 7, 278 (2002).

15) Song K. H., Chung S. J., Shim C. K., J. Control. Rel., 84, 27-37 (2002).

16) Payne N. I., Browning I., Hynes C. A., J. Pharm. Sci., 75, 330-333 (1986).

17) Fang J. Y., Hong C. T., Chiu W. T., Wang Y. Y., Int. J. Pharm., 219, $61-72(2001)$. 